\title{
Bio-inspired, Topologically Connected Colloidal Arrays via Wrinkle and Plasma Processing
}

\author{
Hiroshi Endo,* Yoshiyuki Mochizuki, Masahiro Tamura, and Takeshi Kawai* \\ Department of Industrial Chemistry, Tokyo University of Science, 1-3 Kagurazaka, Shinjuku, Tokyo \\ 162-8601, Japan \\ * To whom correspondence should be addressed \\ Tel: +81-3-5228-8312, Fax: +81-5261-4631. \\ E-mail: endo@ci.kagu.tus.ac.jp, kawai@ci.kagu.tus.ac.jp
}

\begin{abstract}
We investigated the fabrication of various topologically patterned 1D arrays of polystyrene colloidal particles, including single, helical, zigzag, triple-line, and random arrays integrated in sinusoidal wrinkle grooves, through simple spin-coating. In an additional processing step, we used the wrinkled substrates as stamps to transfer the formed polystyrene patterns onto planar supports. Subsequent plasma etching of the particle strings yielded anisotropic, topologically connected particles. Interestingly, these colloidal chains can be easily released and dispersed to maintain the topological alignment and long length $(>100 \mu \mathrm{m})$ with free-standing capability. Depending on the topological structures, these connected particle arrays would enable us to create new bio-inspired materials and devices such as artificial muscles and soft robotics with flexible motion.
\end{abstract}

\section{Keywords}

wrinkle, colloidal assembly, bioinspired, plasma etching, free-standing

(C) 2013. This manuscript version is made available under the Elsevier user license http://www.elsevier.com/open-access/userlicense/1.0/ 


\section{Introduction}

Mother nature provides the ultimate inspiration for various topologically ordered patterns, structures, and flexible motion from one-dimensional (1D) linear structures such as actin filaments and muscle fibers, two-dimensional (2D) arrayed compound eyes of insects, Morpho butterfly wings composed of three-dimensional (3D) hierarchical complex structures, etc. With self-assembly and self-organization, which are the driving principles in the formation of these natural structures, a number of biologically inspired artificial materials have been created [1-4].

Similarly, dimensionally controllable arrays of colloidal particles, from 1D to 2D and 3D colloidal crystals, have attracted much attention because of their potential for many applications in fields ranging from optical and electronic devices to biological and chemical sensors [5-10]. In particular, anisotropic linear arrangements have been greatly anticipated for various fields including energy conversion, surface patterning, coatings, etc. [11-13]. For the most part, two types of approach to self-assembly, such as template-free and template-assisted processes, have been proposed for the fabrication of an anisotropic 1D particle array. Using the template-free self-assembling process [14-23], Watanabe and co-workers obtained spontaneous striped patterns of various colloids on flat substrates by vertical-deposition convective self-assembly [14-17]. Lin and co-workers reported a simple method to assemble latex nanoparticles into regular striped patterns (i.e., coffee-ring-like deposits) over large areas by allowing a drop of an aqueous solution of polystyrene (PS) nanoparticles to evaporate from a liquid capillary bridge, which was formed when the solution was confined between a cylindrical lens and a Si substrate (i.e., cylinder-on-flat geometry) [18]. Ressier and co-workers fabricated a strain sensor consisting of colloidal nanoparticles assembled in wire arrays on a flexible substrate using stop-and-go convective self-assembly (SG-CSA] [19-22]. Similarly, Mori and co-workers proposed a quick fabrication method for striped films using silica arrays and the convective self-assembly technique based on a horizontal coating [23]. However, these 1D particle arrays were mainly arranged into hexagonal or random colloidal aggregates, whose arrangement did not consist of single particle arrays or topologically controlled complex arrays.

On the other hand, template-assisted colloidal assembly under physical confinement [24-31] offers various types of arrangement of colloidal particles, which are difficult to fabricate using template-free approaches. Sow and co-workers reported an effective method for size-selective positioning of colloidal particles on a topographically pre-patterned photoresist surface. The template was fabricated using a direct-laser-writing technique [24]. Yoon and co-workers discovered that by rubbing dry spherical colloidal particles into patterned nanowell arrays, the spherical colloidal particles were very quickly organized into large and perfect 1D arrays [25]. Meseguer and co-workers analyzed the influence of patterned surfaces on the formation of 1D colloidal crystals using a digital versatile disc (DVD) for template-based surface processing [26]. Xia and co-workers obtained 1D zigzag colloidal aggregates under physical confinement by channels etched in a thin film of 
photoresist that was spin-coated on the surface of a glass substrate [27-30]. Kumacheva and co-workers used electrodeposition of monodispersed charged colloidal particles onto an indium-tin-oxide (ITO) substrate patterned with an array of electroconductive grooves, whose progressively diminishing width was commensurate or incommensurate with the dimensions of a discrete number of colloidal particles [31]. While these forms of template-assisted self-assembly show impressive control over positional order and provide directions for the design of complex structures, the template structure is usually produced by top-down approaches such as photolithography, extreme-UV interference lithography, e-beam lithography (EBL), focused-ion-beam (FIB) etching, etc. The employed processes are rather expensive, time- and energy-consuming, and multiple steps are required.

In another approach, colloidal patterning methods in static solution systems [32] or dry processes $[33,34]$, without using template molds or grooves, have been developed to prepare particle wires and strings. However, since most of the aforementioned colloidal structures are formed on rigid substrates such as $\mathrm{Si}$ or glass, the methods have limited use in practical applications. Therefore, there is a demand for straightforward fabrication of flexible templates with design features that can be easily varied from nanoscale to microscale.

Surface wrinkling is an inventive and unconventional technique that is also fast and inexpensive for various types of surface patterning involving sinusoids (ripples), herringbones, labyrinthine designs, etc. It is especially suited for large-area surfaces of poly(dimethylsiloxane) (PDMS) elastomers based on mechanical (buckling) instability [35-37]. This self-organization buckling phenomenon is widely observed in natural systems such as human skin, brain cortex, fruits, and plants $[38,39]$. Owing to the periodic structure and dynamically tunable wrinkles, it has been used in many applications including diffraction gratings [40], stretchable electronics [41,42], antifouling [43], microcontact printing [44], and switching of surface wettability [45,46]. In addition to spherical and inorganic particles [47-54], various materials such as tobacco mosaic viruses (TMV] [55,56], liquid crystals [57,58], and human embryonic stem cell (hESC)-derived cardiomyocytes [59] have been organized (or aligned) into wrinkle grooves (or on surfaces) through dip-coating, spin-coating, and blade processing. In particular, Fery's group demonstrated outstanding results of aligned, 1D gold nanoparticles and poly-N-isopropylacrylamide (pNIPAM)-coated nanostars prepared from their organization into wrinkle grooves, which showed effective surface-enhanced Raman scattering (SERS) platform those. [60,61]

Herein, we describe the fabrication of various topological 1D colloidal arrays, including single, helical, zigzag, triple-line, and random arrays integrated in sinusoidal wrinkle grooves, through simple spin-coating. Moreover, the particles in these arrays can be connected using plasma etching, forming beaded, robust, and long (>100 $\mu \mathrm{m})$ colloidal chains. Interestingly, these colloidal chains can be easily released and dispersed to maintain the topological alignment and length in a free-standing 
state. Depending on the topological structures, these connected particle arrays would enable us to create new bioinspired materials and devices such as artificial muscles and soft robotics with motion.

\section{Experimental}

\subsection{Materials}

Styrene (Kanto Chemicals, Japan) was purified by distillation under reduced pressure in a nitrogen atmosphere. Reagent-grade potassium persulfate (KPS) was used as an initiator without further purification. PDMS was obtained in a Sylgard 184 elastomer kit from Toray Dow Corning, Japan, which consisted of Sylgard 184 monomer and Sylgard 184 base. Polyvinyl alcohol (PVA; $\left.M_{\mathrm{w}} \approx 2,000\right)$ was purchased from TCI, Japan.

\subsection{Synthesis of polystyrene particles}

Monodispersed PS particles with a mean diameter of $560 \mathrm{~nm}$ were synthesized by emulsifier-free polymerization with KPS as the initiator in water $[62,63]$. Styrene monomer (15 g) and water $(180 \mathrm{~mL})$ were added to a $300-\mathrm{mL}$ glass reactor, and the temperature was raised to $75^{\circ} \mathrm{C}$ under vigorous stirring. An aqueous solution $(20 \mathrm{~mL})$ of KPS $(0.35 \mathrm{~g})$ was added to the reactor.

\subsection{Preparation of wrinkles}

PDMS was prepared by mixing the monomer with a base in a weight ratio of 10:1. The mixture was poured into a clean planar petri dish, and the 1-mm-thick film was cured for $2 \mathrm{~h}$ at $65^{\circ} \mathrm{C}$ after degassing overnight under ambient conditions. The crosslinked PDMS was used as the substrate and cut into rectangular pieces with dimensions of $1 \mathrm{~cm} \times 2 \mathrm{~cm}$. To form the surface pattern, the substrate was clamped in a custom-made stretching apparatus and uniaxially stretched until it was $15 \%$ longer than its original length. The PDMS was subsequently oxidized in this pre-strained state using a PIB-20 Plasma Ion Bombarder (Vacuum Device, Japan) at a controllable power level (discharge current) for an adjustable exposure period, and periodic wrinkles were formed perpendicular to the stretching direction once the strain was released. In this study, we prepared four types of wrinkle substrates: (A) approximate wavelength of $1.3 \mu \mathrm{m}$ and amplitude of $220 \mathrm{~nm}$ (7 mA, $5 \mathrm{~min}$ ); (B) approximate wavelength of $2.3 \mu \mathrm{m}$ and amplitude of $400 \mathrm{~nm}$ (7 mA, $25 \mathrm{~min}$ ); (C) approximate wavelength of $3.8 \mu \mathrm{m}$ and amplitude of $660 \mathrm{~nm}$ (40 mA, $10 \mathrm{~min}$ ); (D) approximate wavelength of $5.0 \mu \mathrm{m}$ and amplitude of $800 \mathrm{~nm}(40 \mathrm{~mA}, 10 \mathrm{~min})$.

\subsection{Preparation of particle arrays and formation of bridges}


PS particles were assembled in the grooves of the wrinkles by spin-coating. To ensure a hydrophilic surface, square wrinkled substrates cut to dimensions of $1 \mathrm{~cm} \times 1 \mathrm{~cm}$ were activated by 3 min of plasma treatment. Immediately afterward, an aqueous PS suspension ( $20 \mu \mathrm{L}, 4.7 \mathrm{wt} \%)$ was spin-coated onto the wrinkles (1500 rpm, $30 \mathrm{~s})$. To connect the colloidal array via bridge formation, plasma etching was performed at $10 \mathrm{~mA}$ for controllable period of exposure time.

\subsection{Preparation of free-standing connected particle arrays}

The PS-particle-filled wrinkles were brought into contact with a PVA-coated (spin-coated as a sacrificial layer) glass substrate covered with an ethanol droplet. No external pressure was applied. The attached substrate stayed in contact until the ethanol evaporated. The wrinkled PDMS film was then carefully lifted off, transferring the PS colloidal particles. After plasma etching was performed to transfer the colloidal array on the PVA substrate for $5 \mathrm{~min}$, the substrate was immersed in water to dissolve the sacrificial PVA layer. After the floating PS colloidal arrays were picked up by a micropipette, the aqueous suspensions including the arrays were finally deposited onto a fresh glass substrate.

\subsection{Characterization}

Atomic force microscopy (AFM, S-image, SII) was performed in tapping mode to characterize the surface topography (wavelength and amplitude) of the self-organized, wrinkled substrates. The particle-decorated substrates were characterized by a scanning electron microscope (SEM; Hitachi S-5000, Japan), and the samples were sputtered with $\mathrm{Pt} / \mathrm{Pd}(3-5 \mathrm{~nm})$ by ion sputtering (Hitachi, E1030, Japan).

\section{Results and discussion}

Fig. 1 illustrates the entire fabrication procedure for 1D connected (bridged) colloidal arrays. First, the polydimethylsiloxane substrate was uniaxially stretched, treated with a plasma to generate a stiff thin layer (consisting of a silica-like $\mathrm{SiO}_{\mathrm{x}}$ hard layer [64]). The applied stress was then released to return the substrate to the original state. During the release of applied stress, the difference in Young's modulus of the rigid top layer and the elastic PDMS substrate underneath induced buckling instabilities [35-37], forming sinusoidal wrinkles (Fig. 1(a)). Next, a polystyrene colloidal suspension was spin-coated onto the wrinkled surface to arrange the PS colloids in the wrinkle grooves through high shear force (Fig. 1(b)). Finally, plasma etching was employed to bridge the neighboring particles, yielding anisotropic, connected, 1D PS colloidal arrays (Fig. 1(c)). 
To achieve the self-organization of PS colloids in the wrinkle grooves, a simple and fast spin-coating method was employed. The rotational speed and time were fixed at $1500 \mathrm{rpm}$ and $30 \mathrm{~s}$, respectively, in all experiments. We used four kinds of wrinkled substrates, denoted by (A), (B), (C), and (D) in Fig. 2. These wrinkle structures had approximately (A) $1.3 \mu \mathrm{m}$ wavelength $(W)$ and 220 $\mathrm{nm}$ amplitude (A), (B) $2.3 \mu \mathrm{m}$ wavelength and $400 \mathrm{~nm}$ amplitude, (C) $3.8 \mu \mathrm{m}$ wavelength and 660 $\mathrm{nm}$ amplitude, and (D) $5.0 \mu \mathrm{m}$ wavelength and $800 \mathrm{~nm}$ amplitude. After an aqueous suspension of PS colloids ( $d=560 \mathrm{~nm}, 4.7 \mathrm{wt} \%)$ was spin-coated on the substrates, several arrangements were observed depending on the size of the wrinkles (Fig. 2(Aa,Ba,Ca,Da)). In the case of wrinkle (A), PS colloids were densely arranged to form single colloidal 1D arrays on almost the entire surface, which might have been caused by prevent shared forces to physical accumulation of two or more line assemblies in shallow grooves with amplitudes below the radius of a PS colloid. On the other hand, three types of arrays-helical liner, zigzag, and triple-line structures-were observed in wrinkle (B), with zigzag arrays being the most abundant. The angle $(\theta)$ between the neighboring colloids in a zigzag structure was changed from $180^{\circ}$ for a single particle array in (A) to $90^{\circ}$ and $60^{\circ}$ corresponding to helical liner and zigzag configurations, respectively, in the planar SEM images. With the increase in wrinkle space, the coverage ratio of the helical liner and zigzag arrangements was decreased drastically, and slightly wider triple-line structures occupied most of wrinkle (C). In fact, the distance between the two outermost lines (center-to-center distance) in a triple-line array was expanded from approximately $890 \mathrm{~nm}$ in wrinkle (B) to $940 \mathrm{~nm}$ in wrinkle (C). In the case of the largest wrinkles, wrinkle (D), mostly random arrays were observed. While the two outermost lines in a triple-line array in wrinkles (B) and (C) were parallel (the colloids were arranged to face each other), the lines in (D) were zigzagged depending on the arrangement of colloids at the center of the array. In other words, colloids at the central positions in triple arrays of wrinkles (B) and (C) formed single arrays, while central colloids in random arrays of wrinkle (D) formed zigzag arrays.

Xia and co-workers demonstrated earlier that capillary forces were at play in spherical colloids assembled by physical confinement into discrete or uniform aggregates with well-defined sizes and shapes [27-30]. By using rectangular, flat-bottomed templates, they revealed that the structure of the microspherical assemblies depended on the ratio of channel width $\left(W_{\mathrm{c}}\right)$ to sphere diameter $\left(D_{\mathrm{s}}\right)$. For example, a $W_{\mathrm{c}} / D_{\mathrm{s}}$ value in the range of 0.9-1.1 allowed the microspheres to assemble into a linear chain of single spheres. However, the relationship could not be directly adapted to our case because the wrinkle grooves had unique sidewall widths that varied sinusoidally. Moreover, compared to grooves with rectangular cross sections, even those with the same depth (amplitude), particles would be easily dislocated from the sinusoidal grooves by centrifugal forces. At any rate, various arrangements of colloidal assembly could be easily created by controlling the size of wrinkle patterns. 
Interestingly, the PS colloidal particles adhered to each other and formed connecting sites after assembly (Fig. 2(b) and Fig. S1). This phenomenon resulted from the breakup of surface hydrophilic moieties or surface polymer layers whose glass-transition temperature $\left(T_{\mathrm{g}}\right)$ was lower than that of the bulk material [65]. Going one step further, we subjected these pre-connected colloidal arrays to plasma etching. Plasma etching has two effects on a colloidal array: first, since particles are uniformly etched from all sides, the overall particle size is reduced while each particle remains at its original position; second, connecting bridges (necks) are formed the points where two neighboring particles were touching. The connection is possible by taking advantage of PS particles melting together at their contact point during plasma processing [66]. Owing to these effects, narrow bridged necks were observed between neighboring particles in all samples after 5 min of plasma etching (Fig. 2(c) and (d)), while the topological arrangement remained unchanged.

\section{<Figure 2>}

The plasma etching time can be altered to systematically control the sizes of these bridges and particles. The relationship between etching time, the diameter of reduced particles, and the size of bridge for a 1D single particle array in wrinkle (A) is presented in Fig. 3(a). The terms $x$ axis and $y$ axis refer to the axes perpendicular and parallel to the wrinkle direction, respectively, along which the sizes of structures were measured. These changes in size were evaluated using SEM images (Fig. 3(b)).

Actually, the sizes along both axes (or diameters) of the particles were gradually reduced with increasing etching time. Although we used monodisperse spherical PS particles with an average diameter of $560 \mathrm{~nm}$, the sizes along each axis of the particles were already different in the initial packing state ( $x$ axis: $560 \mathrm{~nm}, y$ axis: $500 \mathrm{~nm}$ ). This was due to the dense packing of neighboring colloids in wrinkle grooves by shear forces and lateral capillary forces [67]. In contrast, the bridge was narrowed and lengthened from about $250 \mathrm{~nm}$ along the $x$ axis and $10 \mathrm{~nm}$ along the $y$ axis in the initial state to $60 \mathrm{~nm}$ and $135 \mathrm{~nm}$, respectively, after $17 \mathrm{~min}$ of etching. When the etching time reached $20 \mathrm{~min}$, the bridge was dissociated and vanished, and the PS particles took on the form of isolated, spherical colloidal dots. After that, the size of these colloidal dots decreased homogeneously and was finally reduced to $160 \mathrm{~nm}$ along both axes. In addition, a semicircular rim resembling a dimple was observed on both sides of the colloidal dot after 20 min of etching, which might have been caused by material molding around the PS particles. This hypothesis was supported by the adhesion observed between the PS colloids and the wrinkled PDMS surface, a result of the deformation of PS colloids, which occurred before the plasma process, owing to plasticization by water or capillary pressure (Fig. S2] [68]. In addition, the plasma process enhanced the thermal deformation. 
Besides reducing the particle size, plasma bombardment also induced roughness on the surface, an effect that has been widely recognized [69]. Indeed, the initial colloidal surface was smooth and clean, while uneven nanoscale structures appeared on the spherical surface after the plasma etching. The surface roughness was higher for a longer etching time than shorter duration owing to microscopically inhomogeneous plasma etching [70,71].

\section{$<$ Figure 3>}

To verify the feasibility of the 1D chains of bridged PS colloids, we released them from the wrinkle grooves. Various colloidal arrays in wrinkles (A)-(D) were first transferred to glass substrates that were each coated with a sacrificial PVA layer by stamping with ethanol (Fig. 1(d)). Plasma etching was then performed for $5 \mathrm{~min}$ to prepare the bridges. It was confirmed that the arrangement of the colloidal particle arrays remained unchanged even after their transfer to PVA-coated substrates and subsequent plasma etching (Fig. S3(a) and (b)). Next, each substrate was immersed in water to dissolve the sacrificial PVA layer. After the floating PS colloidal arrays were picked up by a micropipette, the aqueous suspension containing the arrays was deposited onto another glass substrate (Fig. 1(e)). As a result, a large number of free-standing, connected, 1D PS arrays with physical flexibility and orderly arrangement, including single (Fig. 4(a) and Fig. S4), zigzag (Fig. 4(b) and (c)), helical (Fig. 4(d)), and triple-line arrays(Fig. 4(e)), could be obtained. Moreover, the length of these free-standing beads was very high, about 10-100 $\mu \mathrm{m}$, compared to other connected colloidal beads [54]. On the other hand, all ordered, pre-connected PS beads collapsed in the case when plasma etching was not employed, leading to the formation of random aggregate structures composed of separated particles (Fig. 4(f)). Therefore, rigid and stable connections established through plasma etching was necessary to maintain ordered, 1D PS bead structures with free-standing capability.

There were concerns that undissolved PVA remained in the arrays. The PVA layer was stacked at the bottom of the PS particle array (Fig. 4(c)), and its thermal adhesion might have been enhanced by plasma exposure. To avoid this phenomenon, we tried to directly release the connected, 1D PS colloidal arrays from the wrinkle substrate (Fig. 1(c)) by ultrasonication, without employing a sacrificial PVA layer. After immersion of the connected single colloidal arrays from wrinkle substrate (A) into water, ultrasonication was employed at $28 \mathrm{~Hz}$ for 5-10 min. As a result, shortened, divided, free-standing chains were obtained on a glass substrate after drop casting of the aqueous dispersion (Fig. S5(a)). Compared to PVA processing, however, it was more difficult to obtain longer $(>100 \mu \mathrm{m})$ chains or a large number of free-standing chains with less than 5 min of ultrasonication. Moreover, ellipsoidal structures were observed on individual PS surfaces in the chains obtained after sonication (Fig. S5(b)), whose shape was a good match to the mold on the 
template used for the wrinkle substrate (Fig. S5(c)). This observation was also supported by the deformation of PS colloids in the PDMS wrinkle grooves, as mentioned earlier.

\section{$<$ Figure 4>}

Interestingly, a folding phenomenon was observed in the case of free-standing, long (over 100 $\mu \mathrm{m}$ ) random arrays that were transferred to a glass substrate (Fig. 5(a)); it was not observed in other free-standing chains. The folded and overlapping beads accidentally formed a grid-like structure (Fig. 5(b) and (c)). Although it is difficult to clearly determine the formation mechanism at the present time, we could find this special phenomenon among 1D PS colloidal arrays held by a PVA layer. These Janus-like, anisotropic colloidal chains consisted of asymmetrical objects, with different chemistry or morphology on each side (in our case, PS on one side and PVA on the other), forming new superstructures [72,73] with unusual movements, such as colloidal soft robotics (colloidal swimmer) [74,75], depending on the nature of various colloids and polymers, and unique topological structures.

\section{$<$ Figure 5>}

\section{Conclusion}

In conclusion, we have successfully developed a lithography-free and simple method for the generation of well-ordered anisotropic 1D assemblies of PS particles over large areas. We observed different types of behavior for the particles arranged in the grooves of wrinkled substrates with respect to the pattern formation. Depending on the dimensions of the underlying wrinkles, lines consisting of single particles as well as helical, zigzag, triple-line, and random structures were observed. Using a procedure consisting of simple printing, plasma etching, and dissolution, we were able to transfer a large number of free-standing, long-range topologically ordered PS arrays onto planar solid substrates. Thus, we demonstrated the feasibility of the preparation of anisotropic PS particles in a straightforward process. This work may serve as the proof of concept for the production of long beaded particles, which may find applications as responsive fibers for artificial muscles and soft colloidal robotics [74,75]. Additional studies in progress include the development of a structure that can act as a colloidal swimmer using the connected PS particles with a magnetic nanoaprticle. The results of these investigations will be described in future reports.

\section{Acknowledgment}

This work was partially supported by Adaptable and Seamless Technology Transfer Program through Target-driven R\&D (A-STEP) from JST. 


\section{Figure captions}

\section{Figure 1}

Schematic illustration of the fabrication of connected PS colloidal arrays. After (a) preparation of the PDMS wrinkle surface, (b) PS colloids are assembled into the wrinkle grooves by spin-coating. (c) Plasma etching is employed to bridge neighboring particles in the PS colloidal arrays. (d) To obtain free-standing, connected colloidal arrays, the PS-particle-filled wrinkles are transferred to a PVA-coated (spin-coated as a sacrificial layer) glass covered with an ethanol droplet. After plasma etching to transfer the colloidal arrays, the substrate is immersed in water to dissolve the sacrificial PVA layer. (e) Finally, the aqueous suspension including the arrays is deposited onto another substrate.

\section{Figure 2}

SEM images of various 1D colloidal arrays (a,b) before and (c,d) after plasma etching of four kinds of wrinkle structures: (A) $1.3 \mu \mathrm{m}$ wavelength and $220 \mathrm{~nm}$ amplitude; (B) $2.3 \mu \mathrm{m}$ wavelength and $400 \mathrm{~nm}$ amplitude; (C) $3.8 \mu \mathrm{m}$ wavelength and $660 \mathrm{~nm}$ amplitude; (D) $5.0 \mu \mathrm{m}$ wavelength and 800 nm amplitude. $W$ and $A$ denote wavelength and amplitude, respectively.

\section{Figure 3}

(a) Reducing size of PS colloids and bridges formed in wrinkle (A) as a function of plasma etching time. The terms $x$ axis and $y$ axis refer to the axes perpendicular and parallel to the wrinkle direction, respectively, along which the sizes of structures were measured. (b) Corresponding SEM images of PS colloidal array as a function of plasma etching time.

\section{Figure 4}

SEM images of various free-standing connected colloidal arrays after they were transferred onto glass substrates: (a) single, (b,c) zigzag, (d) helical, and (e) triple-line arrays. (f) PS aggregate structure obtained without plasma etching.

\section{Figure 5}

SEM images of free-standing, connected, random colloidal arrays after they were transferred onto glass substrates. 


\section{References}

(1) Xia, F.; Jiang, L. Adv. Mater. 2008, 20, 2842-2858.

(2) Prouzet, E.; Ravaine, S.; Sanchezd, C.; Backovc, R. New J. Chem. 2008, 32, 1284-1299.

(3) Zhang, T.; Ma, Y.; Qi, L. J. Mater. Chem. B, 2013, 1, 251-264.

(4) Grinthal, A.; Kang, S. H.; Epstein, A. K.; Aizenberg, M.; Khan, M.; Aizenberg, J. Nano Today 2011, 7, 35-52.

(5) Masuda, Y.; Itoh, T.; Koumoto, K. Adv. Mater. 2005, 17, 841-845.

(6) Ye, X.; Qi, L. Nano Today 2011, 6, 608-631.

(7) Li, F.; Josephson, D. P.; Stein A. Angew. Chem. Int. Ed. 2011, 50, 360-388.

(8) Vogel, N.; Weiss, C. K.; Landfester, K. Soft Matter 2012, 8, 4044-4061.

(9) Li, Y.; Cai, W.; Duan G. Chem. Mater. 2008, 20, 615-624.

(10) Kraus, T.; Brodoceanu, D.; Pazos-Perez, N.; Fery. A. Adv. Funct. Mater. 2013, 23, 4529-4541.

(11) Reineck, P.; Lee, G. P.; Brick, D.; Karg, M.; Mulvaney, P.; Bach, U. Adv. Mater. 2012, 24, 4750-4755.

(12) Cheng, J. Y.; Rettner, C. T.; Sanders, D. P.; Kim, H. C.; Hinsberg, W. D. Adv. Mater. 2008, 20, 3155-3158.

(13) Srinivasan, S.; Praveen,V. K.; Philip, R.; Ajayaghosh, A. Angew. Chem. 2008, 120, 5834-5838.

(14) Watanabe, S.; Inukai, K.; Mizuta, S.; Miyahara, M. T. Langmuir 2009, 7287-7295.

(15) Mino, Y.; Watanabe, S.; Miyahara, M. T. Langmuir 2011, 27, 5290-5295.

(16) Watanabe, S.; Mino, Y.; Ichikawa, Y.; Miyahara, M. T. Langmuir 2012, 28, 12982-12988.

(17) Mino, Y.; Watanabe, S.; Miyahara, M. T. ACS Appl. Mater. Interfaces 2012, 4, 3184-3190.

(18) Han, W.; Byun, M.; Lin, Z. J. Mater. Chem., 2011, 21, 16968-16972.

(19) Farcau, C.; Moreira, H.; Viallet, B.; Grisolia, J.; Ressier, L. ACS Nano 2010, 4, 7275-7282.

(20) Farcau, C.; Sangeetha, N. M.; Moreira, H.; Viallet, B.; Grisolia, J.; Ciuculescu-Pradines, D.; Ressier, L. ACS Nano 2011, 5, 7137-7143.

(21) Farcau, C.; Moreira, H.; Viallet, B.; Grisolia, J.; Ciuculescu-Pradines, D.; Amiens, C.; Ressier, L. J. Phys. Chem. C 2011, 115, 14494-14499.

(22) Sangeetha, N. M.; Decorde, N.; Viallet, B.; Viau, G.; Ressier, L. J. Phys. Chem. C 2013, 117, 1935-1940.

(23) Sakamoto, R.; Hataguchi, Y.; Kimura, R.; Tsuchiya, K.; Mori, Y. Chem. Lett. 2012, 41, 12071209.

(24) Varghese, B.; Cheong, F. C.; Sindhu, S.; Yu, T.; Li, C.-T.; Valiyaveettil, S.; Sow, C.-H. Langmuir 2006, 22, 8248-8252.

(25) Khanh, N. N.; Yoon, K. B. J. Am. Chem. Soc., 2009, 131, 14228-14230.

(26) Ramiro-Manzano, F.; Bonet, E.; Rodriguez, I.; Meseguer, F. Langmuir 2010, 26, 4559-4562. 
(27) Xia, Y.; Yin, Y.; Lu, Y.; McLellan, J. Adv. Funct. Mater. 2003, 13, 907-918.

(28) Yin, Y.; Lu, Y.; Gates, B.; Xia, Y. J. Am. Chem. Soc. 2001, 123, 8718-8729.

(29) Yin, Y.; Lu, Y.; Xia, Y. J. Mater. Chem. 2001, 11, 987-989.

(30) Yin, Y.; Xia, Y. J. Am. Chem. Soc. 2003, 125, 2048-2049.

(31) Kumacheva, E.; Golding, R. K.; Allard, M.; Sargent, E. H. Adv. Mater. 2002, 14, 221-224.

(32) Masuda, Y.; Itoh, M.; Yonezawa, T.; Koumoto, K. Langmuir 2002, 18, 4155-4159.

(33) Masuda, Y.; Tomimoto, K.; Koumoto, K. Langmuir 2003, 19, 5179-5183.

(34) Imura, Y.; Kawakami, M.; Morita, C.; Kawai, T. Coll. Surf., A 2010, 358, 153-157.

(35) Vaziri, A. Soft Matter 2010, 6, 5647-5648.

(36) Cerda, E.; Ravi-Chanda, K.; Mahadevan, L. Nature 2002, 419, 579-580.

(37) Bowden, N.; Brittain, S.; Evans, A. G.; Hutchinson, J. W.; Whitesides, G. M. Nature 1998, 393, 146-149.

(38) Chen, X.; Yin, J. Soft Matter, 2010, 6, 5667-5680.

(39) Li, B.; Cao, Y.-P.; Feng, X.-Q.; Gao, H. Soft Matter, 2012, 8, 5728-5745.

(40) Harrison, C.; Stafford, C. M.; Zhang, W. H.; Karim, A. Appl. Phys. Lett. 2004, 85, 4016-4018.

(41) Wagner, S.; Lacour, S. P.; Jones, J.; Hsu, P. H. I.; Sturm, J. C.; Li, T.; Suo, Z. G. Phys. E 2004, 25, 326-334.

(42) Khang, D. Y.; Jiang, H. Q.; Huang, Y.; Rogers, J. A. Science 2006, 311, 208-212.

(43) Hoipkemeier-Wilson, L.; Schumacher, J.; Carman, M.; Gibson, A.; Feinberg, A.; Callow, M.; Finlay, J.; Callow, J.; Brennan, A. Biofouling 2004, 20, 53-63.

(44) Meitl, M. A.; Zhu, Z. T.; Kumar, V.; Lee, K. J.; Fen, X.; Huang, Y. Y.; Adesida, I.; Nuzzo, R. G.; Rogers, J. A. Nat. Mater. 2006, 5, 33-38.

(45) Lin, P. C.; Yang, S. Soft Matter 2009, 5, 1011-1018.

(46) Zhao, S.; Xia, H.; Wu, D.; Lv, C.; Chen, Q.-D.; Ariga, K.; Liu, L.-Q.; Sun, H.-B. Soft Matter 2013, 9, 4236-4240.

(47) Lu, C.; Möhwald, H.; Fery, A. Soft Matter 2007, 3, 1530-1536.

(48) Schweikart, A.; Fortini, A.; Wittemann, A.; Schmidt, M.; Fery, A. Soft Matter 2010, 6, 5860-5863.

(49) Schweikart, A.; Pazos-Pérez, N.; Alvarez-Puebl, R. A.; Fery, A. Soft Matter 2011, 7, 4093-4100.

(50) Müller, M; Karg, M.; Fortini, A.; Hellwegd, T.; Fery A. Nanoscale 2012, 4, 2491-2499.

(51) Badre, C.; Chapel, J. P.; Yang, S. Soft Matter 2011, 7, 9886-9889.

(52) Hyun, D. C.; Moon, G. D.; Cho, E. C.; Jeong, U. Adv. Funct. Mater. 2009, 19, 2155-2162.

(53) Hiltl, S.; Oltmanns, J. Böker, A. Nanoscale 2012, 4, 7338-7345.

(54) Hiltl, S. Schürings, M.-P.; Balaceanu, A.; Mayorga,V.; Liedel, C.; Pich A.; Böker, A. Soft Matter 2011, 7, 8231-8238. 
(55) Horn, A.; Hiltl, S.; Fery, A.; Böker, A. Small 2010, 6, 2122-2125.

(56) Horn, A.; Schoberth, H.; Hiltl, S.; Chiche, A.; Wang, Q.; Schweikart, A.; Fery, A.; Böker, A. Faraday Discuss. 2009, 143, 143-150.

(57) Ohzono, T.; Monobe, H. Yamaguchi, R.; Shimizu, Y.; Yokoyama, H. Appl. Phys. Lett. 2009, $95,014101$.

(58) Ohzono, T.; Fukuda, J. Nat. Commun. 2012, 3, 701, DOI:10.1038/ncomms1709.

(59) Chen, A.; Lieu, D. K.; Freschauf, L.; Lew, V.; Sharma, H.; Wang, J.; Nguyen, D.; Karakikes, I.; Hajjar, R. J.; Gopinathan, A.; Botvinick, E.; Fowlkes, C. C.; Li, R. A.; Khine, M. Adv. Mater. 2011, 23, 5785-5791.

(60) Pazos-Pérez, N.; Ni, W.; Schweikart, A.; Alvarez-Puebl, R. A.; Fery, A.; Liz-MarzánL, L. M. Chem. Sci., 2010, 1, 174-178.

(61) (b) Müller, M; Tebbe, M.; Andreeva, D. V.; Karg, M.; Alvarez-Puebl, R. A.; Pazos-Pérez, N.; Fery, A. Langmuir 2012, 28, 9168-9173.

(62) Okubo, M.; Izumi, J. Colloids Surf. A 1999, 153, 297-304.

(63) Kawai, T.; Suzuki, M.; Kondo, T. Langmuir 2006, 22, 9957-9961.

(64) Bowden, N.; Huck, W. T.; Paul, K. E.; Whitesides, G. M. Appl. Phys. Lett. 1999, 75, 25572559.

(65) Yabu, H. Langmuir 2013, 29, 1005-1009.

(66) Lee, B.-K.; Kim, K. S.; Lee, J.-H.; Kim, N.-H.; Roh, Y. J. Vac. Sci. Technol. A 2008, 26, 819-823.

(67) Dimitrov, A. S.; Nagayama, K. Langmuir 1996, 12, 1303-1311.

(68) Jurewicz, I.; Keddie, J. L. Dalton, A. B. Langmuir 2012, 28, 8266-8274.

(69) Zhao, Y. P.; Drotar J. T.; Wang G. C.; Lu, T. M. Phys. Rev. Lett. 1999, 82, 4882-4885.

(70) Shiu, J. Y.; Kuo, C. W.; Chen, P. L.; Mou, C. Y. Chem. Mater. 2004, 16, 561-564.

(71) Haginoya, C.; Ishibashi, M.; Koike, K. Appl. Phys. Lett. 1997, 71, 2934-2936.

(72) Walther, A.; Muller, A. H. E. Soft Matter 2008, 4, 663-668.

(73) Perro, A.; Reculusa, S.; Ravaine, S.; Lami, E. B.; Duguet, E. J. Mater. Chem. 2005, 15, 3745-3760.

(74) Snezhko, A.; Aranson, I. S. Nat. Mater. 2011, 10, 698-703.

(75) Tierno, P.; Golestanian, R.; Pagonabarraga, I.; Sagués. F. J. Phys. Chem. B 2008, 112, $16525-16528$. 
Figure 1

(a)

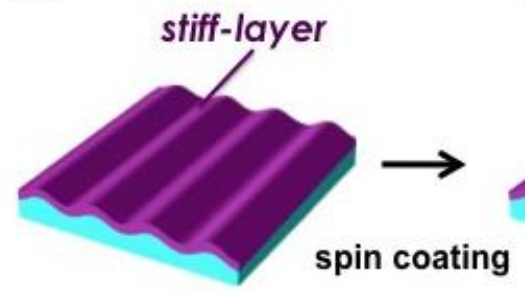

wrinkled-PDMS (b)

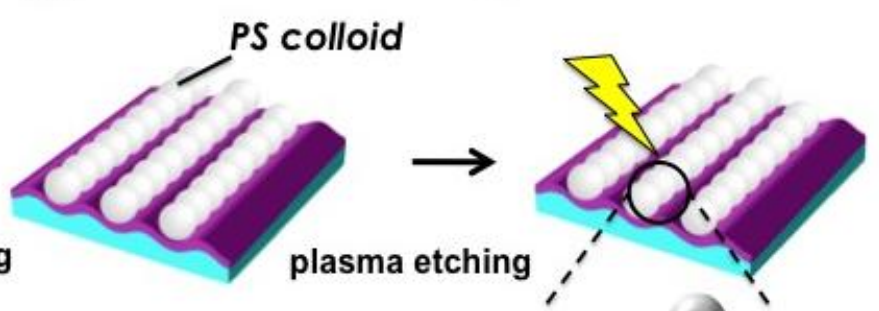

(d) $\downarrow$ transfer

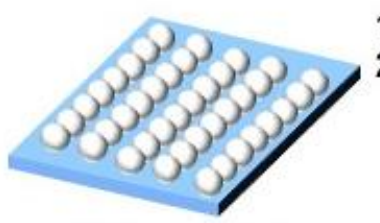

PVA-coated substrate (c)

(e) bridge formation

1) plasma

2) remove

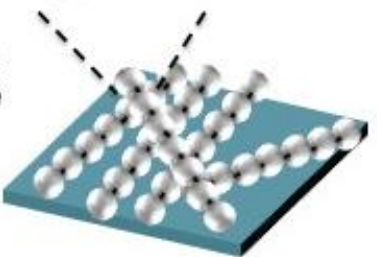

Free-standing connected colloidal chain 
Figure 2
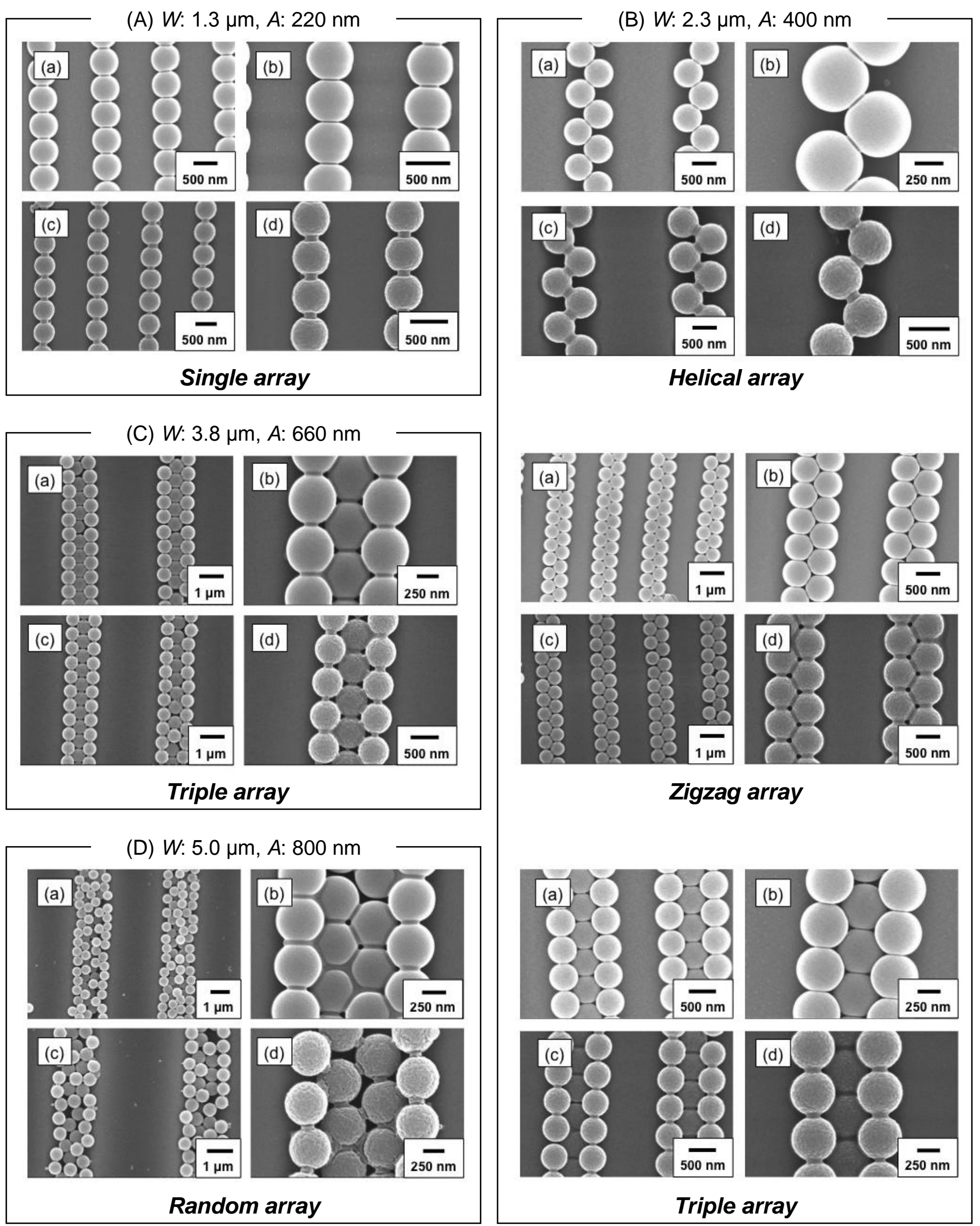
Figure 3

(a)

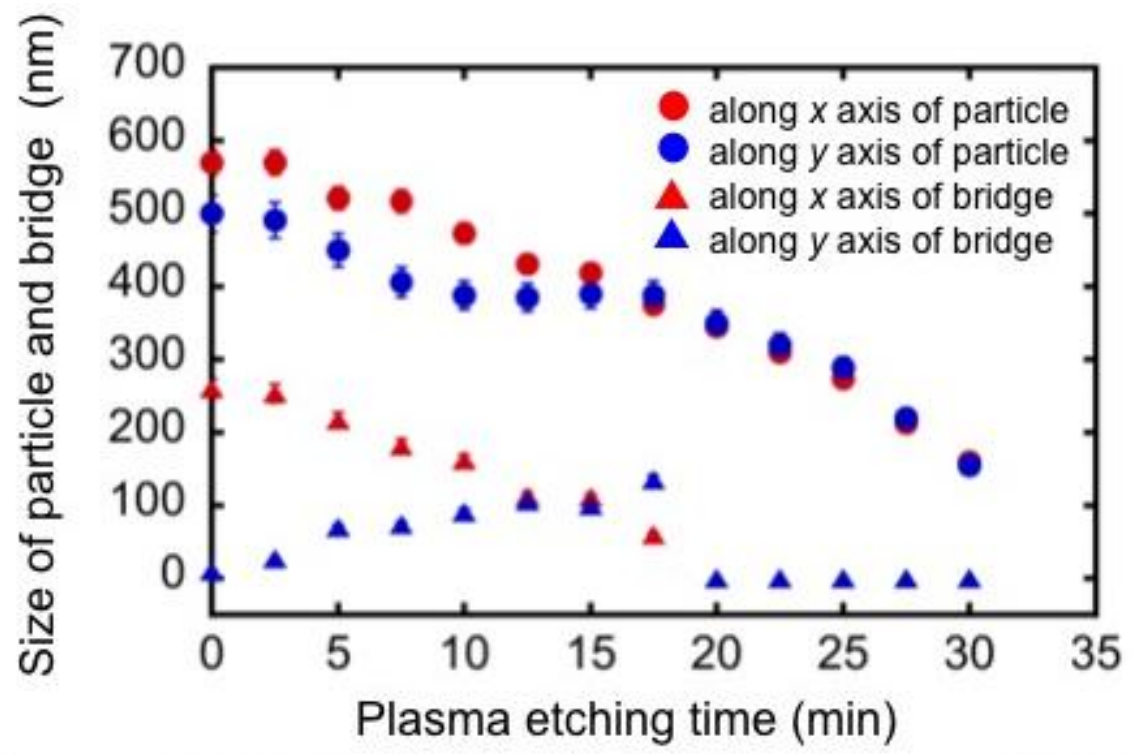

(b)
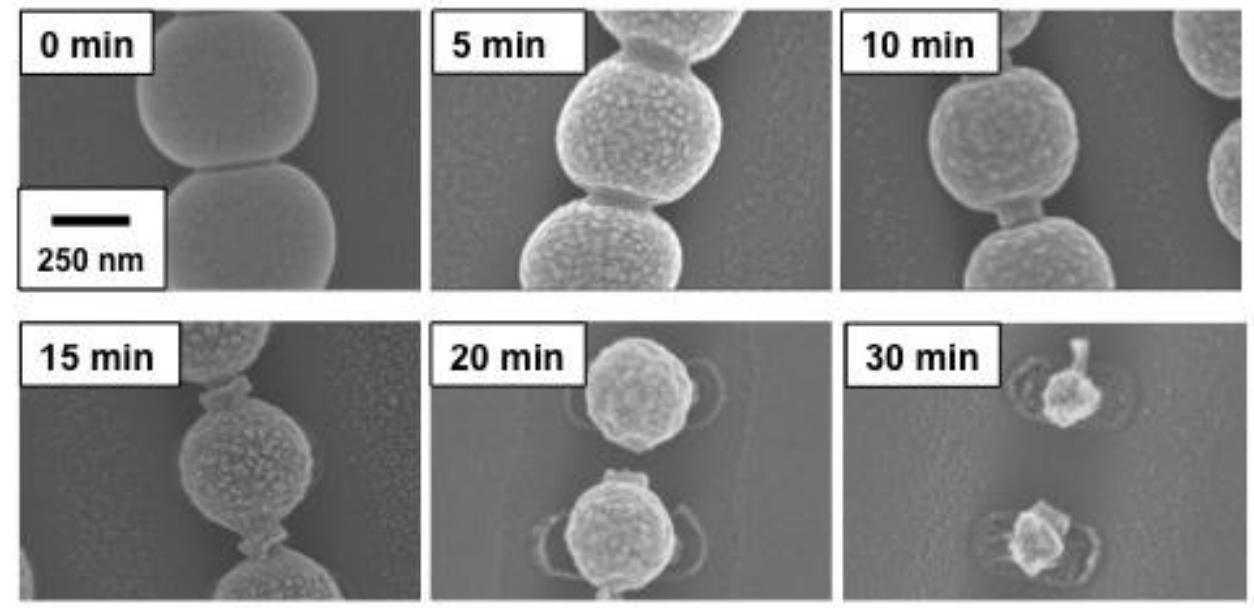
Figure 4
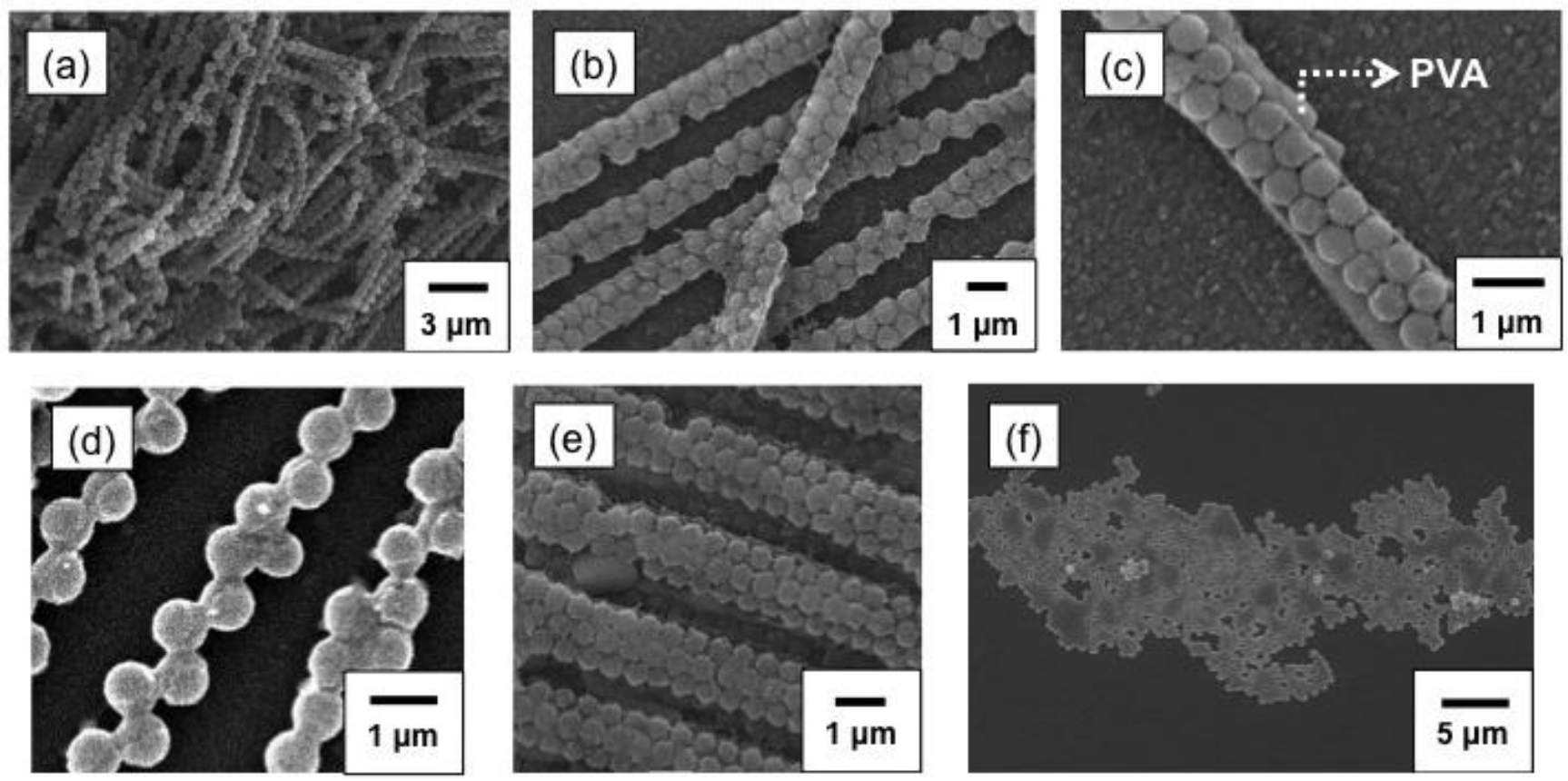
Figure 5
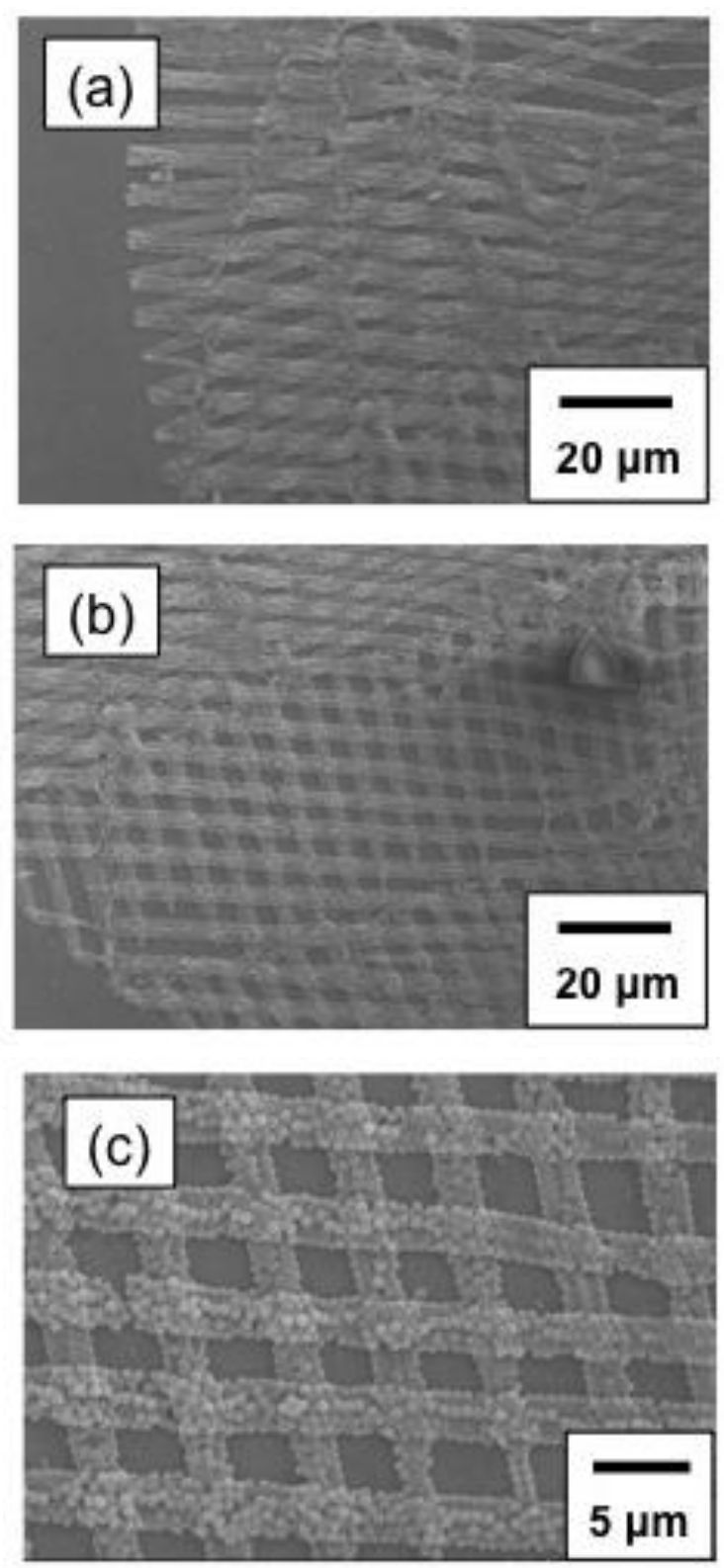
Graphical Abstract

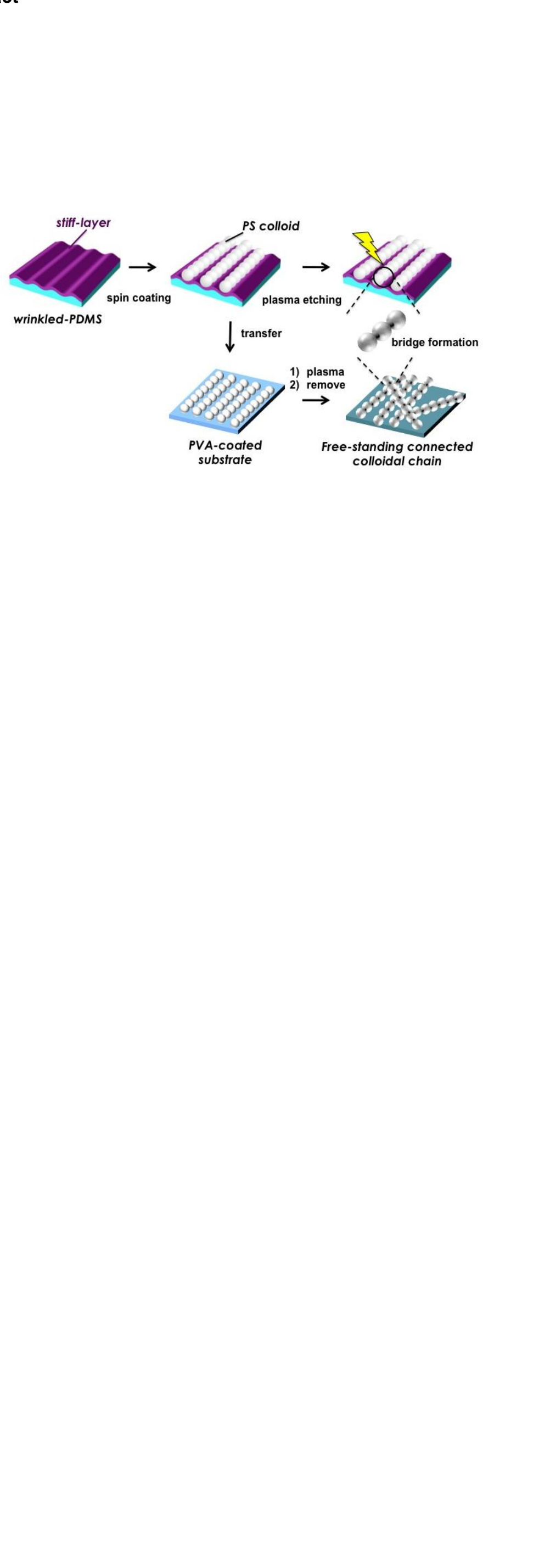

.

\author{
.
}

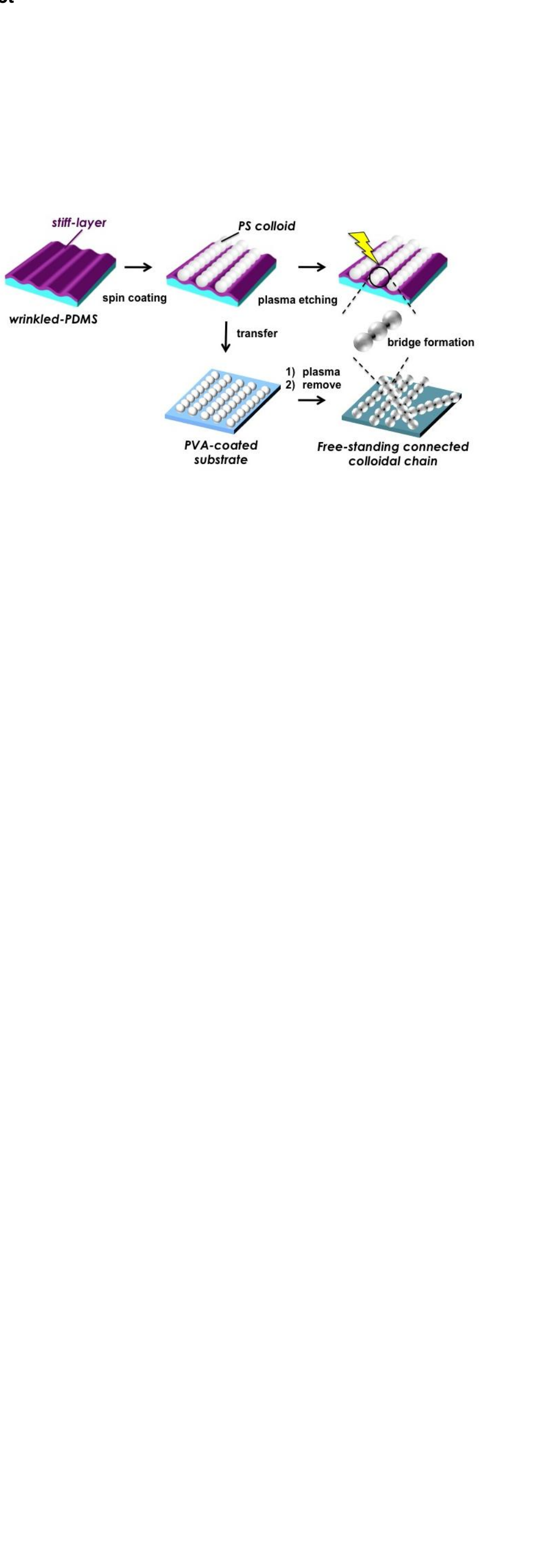
.

.

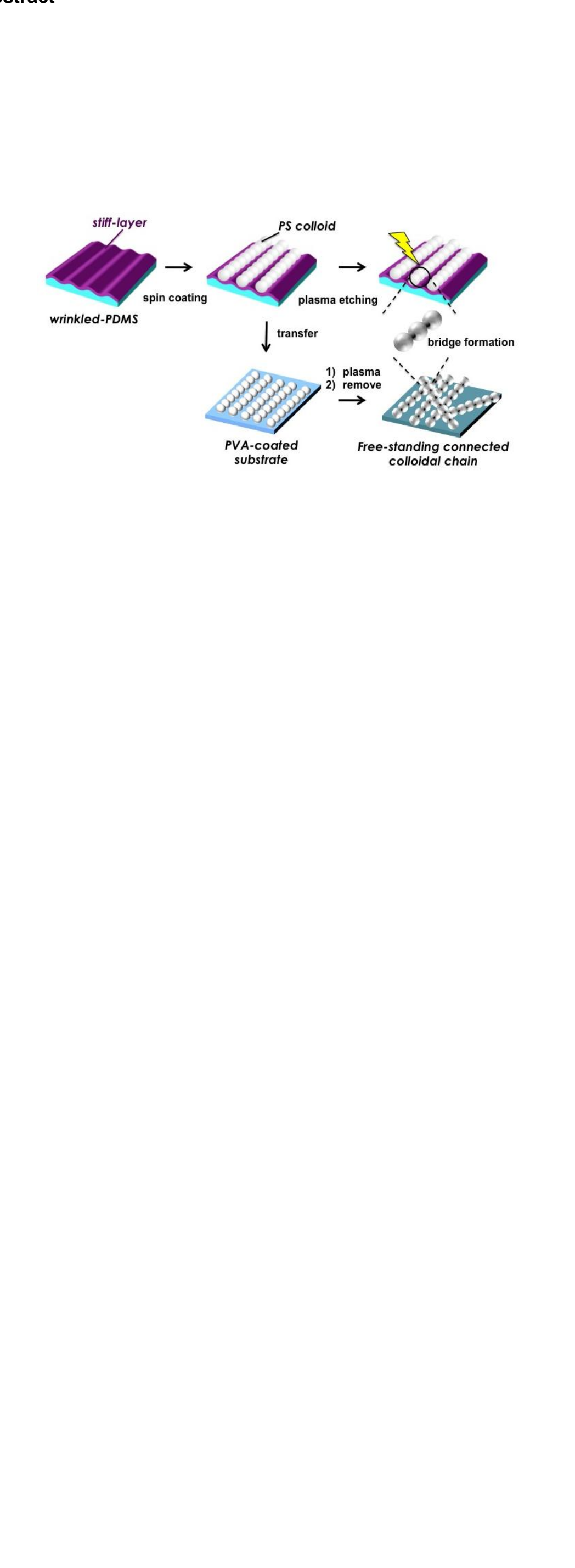

